\title{
Gamma bands in doubly odd rhenium and iridium nuclei
}

\author{
M. Balodis ${ }^{1, a}$, J. Bērzinš ${ }^{1, b}$, T. Krasta ${ }^{1, c}$, and L. Simonova ${ }^{1}$ \\ ${ }^{1}$ Institute of Solid State Physics, University of Latvia, LV-1063, Riga, Latvia
}

\begin{abstract}
Structure of the $|K \pm 2|$ bands in doubly-odd nuclei belonging to the transitional deformation region at A 190 is discussed. Relation of these quasi gamma-bands with the non-axial deformation of the parent twoquasiparticle configurations is studied. Using available experimental information, new tentative $|K \pm 2|$ bands are proposed in ${ }^{188} \mathrm{Re}$, and ${ }^{192,194} \mathrm{Ir}$ nuclei. Coexistence of two-quasiparticle states with different deformation modes is considered in the case of ${ }^{188} \mathrm{Re}$ and ${ }^{194} \mathrm{Ir}$.
\end{abstract}

\section{Introduction}

Nuclei of the transitional A $\sim 190$ region are characterized by core instability. Shape phase transitions in the case of even-even nuclei have been a theme of extensive theoretical studies. Attempts have been made to extend these studies also to odd-A nuclei and it has been shown that the phase-transitional behaviour of the even-even core is retained when one adds an additional fermion. For doublyodd nuclei, studies of nuclear shape transitional phenomena are hindered, first, by the complexity of theoretical description, and, second, by the lack of experimental data related with collective core excitations.

In the case of non-axial nuclear shape for each value of the total nuclear momentum $I$, one obtains $2 I+1$ levels with $I$ projection $K$ assuming value in the range $-I,-I+1, \ldots, 0, \ldots I-1, I$. As a result, one observes a number of $|K \pm 2|,|K \pm 4|, \ldots$ side-bands for each twoquasiparticle configuration. Characteristic feature of these bands is intense decay to the levels of its parent $K$ band, predominantly with $\mathrm{E} 2$, or $\mathrm{M} 1+\mathrm{E} 2$ transitions.

One should note that establishing of gamma-bands in odd-odd nuclei is a disputable theme. Because of the complexity of experimental data related with the development of odd-odd nucleus level scheme, structure of these nuclei is not so well studied as in the case of their even-even, and odd-A neighbours. As a result, one can rarely expect to obtain confident direct proof for the collective nature of the band in terms of experimental $B(E 2)$ values for depopulating transitions.

However, there are several usable indirect indications: a) proposed gamma-band depopulates most intensely to the levels of its parent configuration band, and depopulating transitions have prominent E2 multipolarity components; b) no two-quasiparticle configuration with given spin/parity values is expected in the investigated energy

\footnotetext{
ae-mail: martins.balodis@latnet.lv

be-mail: jberzins@latnet.lv

ce-mail: krasta@latnet.lv
}

range; c) gamma-bands in neighbouring even-even and odd-A nuclei provide information about expected core $E_{2+}$ energy; d) rotational parameters of the proposed gammaband are close to those of the parent band.

In the presented study, we have tried to identify lowest $|K \pm 2|$ collective excitations of the nuclear core in some doubly-odd rhenium and iridium nuclei. For theoretical structure calculations, we have used the two-quasiparticles plus asymmetric rotor model $[1,2]$.

\section{The $|K \pm 2|$ bands in doubly-odd rhenium and iridium nuclei}

The best established gamma bands in doubly odd nuclei with $\mathrm{A} \sim 190$ are: the $351.6 \mathrm{keV} 2^{+}|K-2|$ band in ${ }^{192} \operatorname{Ir}[3$, 4], based on the $K^{\pi}=4^{+}$(p:11/2[505]-n:3/2[512]) ground state configuration strongly mixed with the (p:3/2[402]$\mathrm{n}: 11 / 2[615])$ configuration, and the $2^{+}|K-2|$ band at 518.6 $\mathrm{keV}$ in ${ }^{194} \mathrm{Ir}$ [5], based on the $147.1 \mathrm{keV} 4^{+}$level with the structure analogous to that of the $4^{+}$ground state in ${ }^{192} \mathrm{Ir}$. Since no experimental $B(E 2)$ values have been measured for transitions depopulating levels of these bands, their interpretation has been based on indirect indications.

Using available experimental data $[3,6]$, we have reconsidered recently the ${ }^{192}$ Ir level scheme proposed in [4]. In the new level scheme, there are more than 10 levels below $530 \mathrm{keV}$ which one cannot interpret as members of some already existing band, or the bandheads of expected two-quasiparticle configurations. We believe that it is a clear indication of triaxiality and three of these new levels $\left(6^{+} 352.9 \mathrm{keV}, 4^{+} 368.2 \mathrm{keV}\right.$, and $\left.4^{-} 380.4 \mathrm{keV}\right)$ can be tentatively associated with the $|K \pm 2|$ core excitations of lowest two-quasiparticle configurations. All three levels have confidently established spins and parities and decay mostly to the levels of corresponding parent configuration. Their energies correspond approximately to the two-quasiparticle state bandhead energy plus energy of the core $2^{+}$state derived from the $351.7 \mathrm{keV} 2^{+}$level energy. 


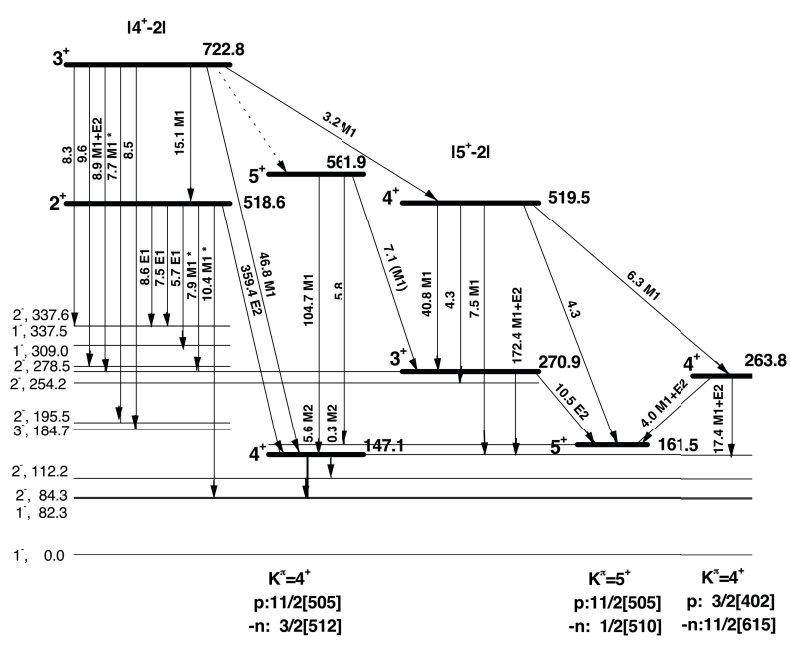

Figure 1. Interpreted positive parity level scheme of ${ }^{194}$ Ir. Experimental data are taken from [5].

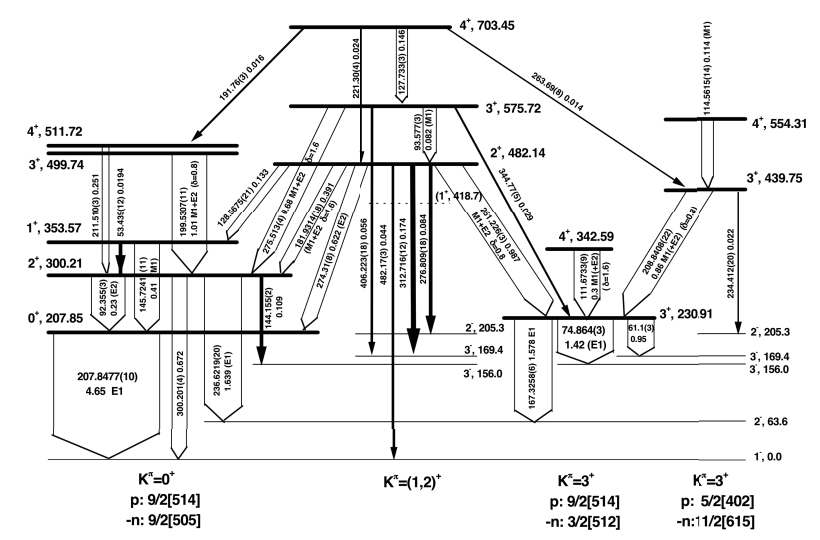

Figure 2. Partial positive parity level scheme of ${ }^{188}$ Re. Experimental data are taken from the manuscript in preparation.

Now, let us consider the neighbouring ${ }^{194}$ Ir nucleus. In [5], the low-lying $3^{+}$rotational band has been established at $270.9 \mathrm{keV}$ (see figure 1). However, the available proton and neutron orbits do not predict $3^{+}$band in this energy region. The $270.9 \mathrm{keV}$ level decays with intense E2 transition to the $161.5 \mathrm{keV} 5^{+}$level, and M1+E2 transition to the $4^{+}$level at $147.1 \mathrm{keV}$. We suggest that the $3^{+} 270.9 \mathrm{keV}$ band can be a $|K-2|$ gamma band of the $K^{\pi}=5^{+}(\mathrm{p}: 11 / 2[505]+\mathrm{n}: 1 / 2[510])$ configuration established at $161.5 \mathrm{keV}$. Though, in such a case, one must consider coexistence of different non-axial shapes in ${ }^{194} \mathrm{Ir}$ since energy of the $2^{+}$core state for the $270.9 \mathrm{keV}$ band is much smaller than that for the $518.6 \mathrm{keV} 2^{+}|K-2|$ band.

Shape coexistence is possible also in ${ }^{188} \mathrm{Re}$. Our study of the ${ }^{188}$ Re levels [7] disclosed some problems for interpretation of the level scheme above $400 \mathrm{keV}$. First, the experimental density of positive parity levels below $1 \mathrm{MeV}$ is about three times higher than predicted by the performed particle plus rotor coupling model (PRC) calculations with the axially-symmetric core deformation. Second, we have not observed population of the $K^{\pi}=1^{+}$(p:9/2[514]$\mathrm{n}: 7 / 2[503])$ bandhead predicted at about $418.7 \mathrm{keV}$.

Depopulation of the $482.1 \mathrm{keV}$ level (see figure 2) indicates it as a $2^{+}$bandhead. The associated rotational band is quite regular: the rotational parameters are $A_{0}=15.12$ $\mathrm{keV}$, and $B=0.026 \mathrm{keV}$ ( $c f ., A_{0}=15.48 \mathrm{keV}$, and $B=-0.014$ $\mathrm{keV}$ for the even spin levels of the $K^{\pi}=0^{+}$band). On the contrary, the $K^{\pi}=1^{+}$(p:9/2[514]-n:7/2[503]) band should display a notable staggering of odd-even spin levels due to strong Coriolis interaction with levels of the $K^{\pi}=0^{+}$ (p:9/2[514]-n:9/2[505]) band.

Since the low-lying proton and neutron orbits do not predict a $2^{+}$bandhead below $700 \mathrm{keV}$ in ${ }^{188} \mathrm{Re}$, we have considered possibility that the $2^{+}$band at $482.1 \mathrm{keV}$ is a $|K+2|$ band of the $K^{\pi}=0^{+}$(p:9/2[514]-n:9/2[505]) configuration. The established decay pattern agrees with such interpretation. The $274.31 \mathrm{keV}$ transition to the $207.9 \mathrm{keV} 0^{+}$level is a part of doublet line resolved by $\gamma \gamma$-coincidences. Therefore, the M1+E2 multipolarity assigned in [8] does not contradict assumption that the $274.31 \mathrm{keV}$ transition has pure E2 multipolarity.

We have performed the PRC model calculations of the ${ }^{188} \mathrm{Re}$ positive parity levels in the case of non-axial core deformation. The theoretical predictions at asymmetry angle $\gamma=20^{\circ}$ show that one obtains bandhead of the $|K+2|$ side-band at about $480 \mathrm{keV}$ energy and the $K^{\pi}=1^{+}$bandhead $\sim 50 \mathrm{keV}$ higher. However, the results of calculations were unsatisfactory for positive parity bands with $K \geq 3$. For these levels, the axially-symmetric PRC model was preferable. Therefore, we assume that the non-axial deformation in ${ }^{188} \mathrm{Re}$ is associated only with the (p:9/2[514]$\mathrm{n}: 9 / 2[505])$ configuration when both proton and neutron occupy high $j$ orbits.

\section{References}

[1] T. Bengtsson, I. Ragnarsson, Nucl. Phys, A 436, 14 (1985)

[2] S. E. Larsson, G. Leander, I. Ragnarsson, Nucl. Phys. A 307, 189 (1978)

[3] J. Kern et al., Nucl. Phys. A 534, 77 (1991)

[4] M. Balodis, In: Proc.9th Int.Symp. on Capture Gamma-Ray Spectroscopy and Related Topics. Eds. G. L. Molnar, T. Belgya and Zs. Revay. Vol.1 (World Scientific, Singapore, 1997), 147

[5] M. Balodis et al., Phys. Rev. C 77, 064602 (2008)

[6] P. E. Garrett, D. G. Burke, Nucl. Phys. A 568, 445 (1994)

[7] M. Balodis et al., Nucl. Phys. A 847, 121 (2010)

[8] A. A. Suarez et al., Nucl. Phys. A 107, 417 (1988) 